\title{
Iris atrophy with hypoperfusion and microneovascularisation
}

\author{
ANNE M V BROOKS AND WILLIAM E GILLIES \\ From the Glaucoma Investigation and Research Unit, Royal Victorian Eye and Ear Hospital, East Melbourne, \\ Victoria 3002, Australia
}

SUMMARY A series of 17 patients with stromal atrophy, hypoperfusion, and microneovascularisation of the iris investigated in the Glaucoma Investigation and Research Unit are described, and their iris angiograms were compared with those of normal irides of patients in the same age group seen in general clinics. In all but one of the 17 cases this iris atrophy was associated with glaucoma or ocular hypertension, which appeared to be secondary to the iris changes. The condition was bilateral and presented a typical slit-lamp appearance, with subtle evidence of microneovascularisation. There was neither history nor clinical evidence of previous trauma, heterochromia, or intraocular inflammation. The commonest form of iris atrophy affected the inner third of the iris stroma in a patchy manner, often with sparing above. However, diffuse atrophy occurred in two cases, and there were two cases of 'senile tears' of the iris. Some accompanying atrophy of the pigment epithelium was usual but less prominent. The changes on fluorescein angiography of the iris included the late appearance of dye with a long arteriovenous circulation time, fewer arteries than normal with sectorial hypoperfusion, leakage of dye from the pupil margin and peripupillary neovascularisation, stromal tufts, and sometimes more complex stromal microneovascularisation. An expanded prominent lesser vascular circle was a common feature of the condition. The condition is bilateral and distinct from other forms of iris atrophy. In all cases the iris changes appeared to be secondary to the vascular hypoperfusion and were not consistently associated with evidence of gross vascular disease. All patients had grey (blue) irides, and this may be an aetiological factor. The condition appears common enough to form a significant group of glaucoma patients and to be a separate clinical entity.

The association of iris anomalies with glaucoma is well recognised. They are commonly seen with congenital and developmental glaucomas,' particularly in familial hypoplasia of the iris, ${ }^{2}$ hypoplasia of the iris due to congenital rubella, ${ }^{3}$ aniridia, ${ }^{+}$and in iridocorneal and irideal dysgenesis as seen in Rieger's syndrome and Peters' anomaly. ${ }^{56}$ In later life essential atrophy of the iris, ${ }^{7 \times}$ Chandlers syndrome," and the iris naevus syndrome (ICE syndrome)" form a group of cases distinct from the entity of iridoschisis." Anterior segment pigment dispersal syn-

Address for reprints: Glaucoma Investigation and Research Unit, Royal Victorian Eye and Ear Hospital, East Melbourne, Victoria, 3002 Australia.

Correspondence to Dr W E Gillies, 82 Collins Strect, Melbourne, Victoria 3000, Australia. drome is associated with changes in the pigment epithelium of the iris, and a recent paper describes anomalies of the iris vasculature in this condition.' Changes in the iris are also present in pseudoexfoliation of the lens capsule associated with glaucoma ${ }^{1:-15}$ and Fuchs' heterochromic cyclitis, ${ }^{1617}$ and atrophy of the iris has long been recognised after acute angle closure glaucoma. ${ }^{1 x}$

We have recently observed a series of cases distinct from the foregoing which show stromal strophy of the iris, iris hypoperfusion, and microneovascularisation. These cases are distinct enough to form a separate clinical entity. They are usually, but not invariably, associated with glaucoma. The purpose of this paper is to describe the findings in detail, consider their relationship to glaucoma, and to speculate on the aetiology of the observed changes. 


\section{Materials and methods}

A series of 17 patients with varying degrees of atrophy of the iris stroma were investigated at the Glaucoma Investigation and Research Unit of the Royal Victorian Eye and Ear Hospital. There were 10 males and seven females with ages ranging from 67 to 89 years (mean 78, SEM 2 years). In all cases the colour of the iris was grey (or blue). Eight patients had chronic open angle glaucoma, seven patients had varying degrees of ocular hypertension with open angles, one had subacute angle closure glaucoma but had not suffered an acute attack of glaucoma, while one patient was discovered to have iris atrophy with normal pressures. Six patients had started treatment for raised intraocular pressure with timolol maleate and pilocarpine hydrochloride only some months before the iris atrophy was noted.

All the patients were examined carefully, and the iris changes and any evidence of pigment epithelial loss were recorded. Contact specular microscopy was performed with the Pocklington contact specular microscope.

All the patients underwent fluorescein angiography of the iris to delineate the iris vascular changes in this condition. Fluorescein iris angiography was performed with a Nikon camera and 105 $\mathrm{mm}$ macro lens attached to the side of the Zeiss fundus angiography camera, utilising the light source and filter; this left the eyepiece of the angiogram camera free for use by an observer. The subjects - received an intravenous bolus injection of $10 \mathrm{ml}$ of $25 \%$ sodium fluorescein.

The time of first appearance of the dye (from its intravenous injection to its appearance in the iris vessels) and the arteriovenous circulation times (the time from when the arteries initially fill until the beginning of the venous phase) were measured, the number of radial arteries was estimated, and sectorial arterial filling defects were noted. Sectorial arterial defects were present when there was failure of a sector to perfuse during the first arterial phase of perfusion and filling occurred only some time after the venous flush had occurred elsewhere. A sectorial arterial defect was distinct from a delay in arterial perfusion; in the latter case the arterioles in a sector filled late in the arterial phase, or even very early in the venous phase. Leakage of dye from abnormal vessels was also recorded.

In view of the finding of iris hypoperfusion, intravenous digital subtraction angiography was performed on 10 patients to exclude internal carotid artery disease, which has been reported in ischaemic ocular inflammation. ${ }^{1 "}$ A non-ionic contrast medium, Iopamira 300 was used, and the catheter tip was placed in the right atrium. This enabled us to examine

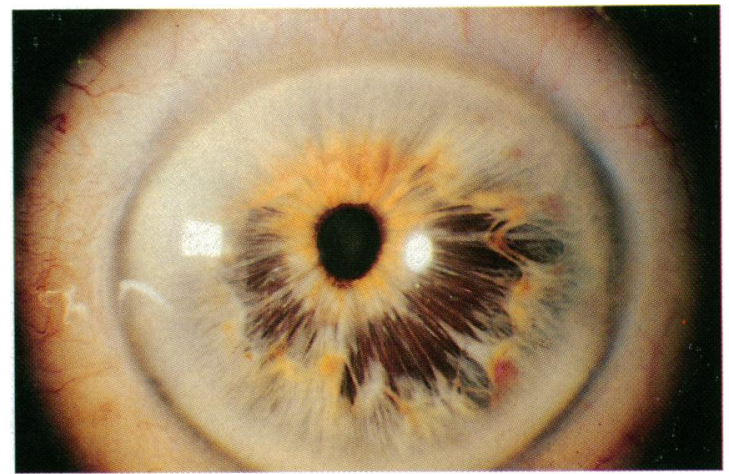

Fig. 1 Well marked atrophy of the inner third of the iris stroma in a male aged 73 years with ocular hypertension. The atrophy does not affect the upper third of the iris stroma and lies almost entirely internal to an expanded lesser vascular circle of the iris.

the carotid bifurcation, aortic arch, intracerebral circulation to the level of the siphon, and the vertebral arteries.

For comparison the iris angiograms of a series of 10 patients, eight females and two males of similar age (range 69 to 85 years, mean 77, SEM 2 years) were examined. The iris appeared normal on slit-lamp examination in all these patients.

\section{Results}

The changes we describe were similar though not equal in degree in each eye of all patients. No patient suffered from diabetes mellitus and intraocular pressure was controlled to within the normal range at the time of angiography. All cases showed atrophy of the iris stroma, and this took several forms:

1. Atrophy of the inner third of the iris stroma internal to the lesser vascular circle of the iris. The lesser vascular circle of the iris then appeared to be expanded, so that in the affected areas it was a greater distance from the pupil margin than in normal subjects. The atrophy was irregular and patchy, often with sparing of the upper third of the iris stroma, so that the lesser vascular circle was also irregular in outline and closer to the pupil above. This form of iris atrophy was the most common type and was present in varying degrees in 14 cases (Figs. 1, 2). The appearance was distinct from the 'shredded wheat' appearance of iridoschisis, where fine strands of 'shredded' stroma float partly free in the anterior chamber.

2. Diffuse atrophy of the iris stroma. There was a generalised loss of the normal crypts and collarette with the appearance of an open, thin sponge-like structure through which the pigment epithelium was 


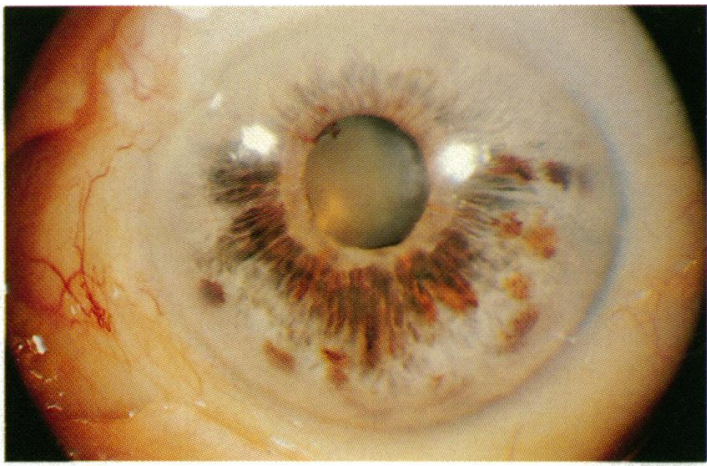

Fig. 2 Marked atrophy of the iris stroma in a female aged 80 years with chronic open angle glaucoma. Below, the atrophy also involves the pigment epithelium of the iris so that there is a through-and-through defect. The upper third of the iris stroma is unaffected. See Fig. 5 for the pigment epithelial loss.

visible. Two cases showed this form of stromal atrophy (Fig. 3).

3. 'Senile tears' of the iris. This unusual finding was bilateral, with the appearance of multiple fine tears at the pupil margin; the iris stroma was particularly involved, but there was also loss of the collarette. There was neither a history nor signs of contusion injury in the anterior segment in these patients. 'Senile tears' were present in two cases (Fig. 4).

An accompanying atrophy of the pigment epithelium of the iris was usually less marked than the stromal atrophy, with the following features:

1. Loss of the pigmented ruff at the pupil margin; this was present in all cases to some degree (Fig. 4).

2. Diffuse atrophy of the pigment epithelium of the iris, so that there was diffuse light reflux through the pigment epithelium on retroillumination. This

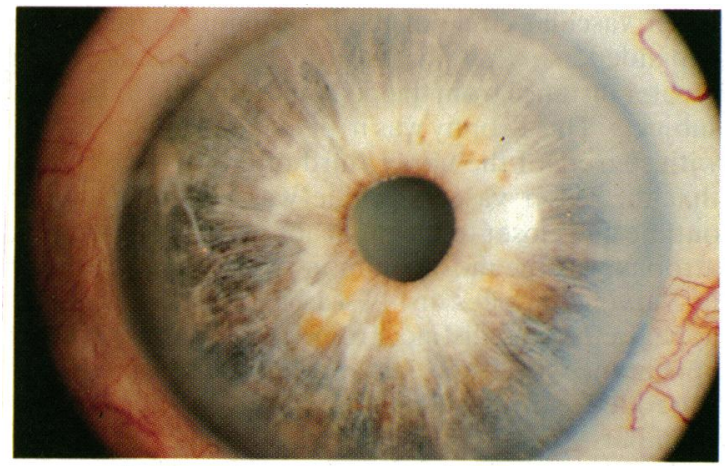

Fig. 3 Diffuse atrophy of the iris stroma so that the pigment epithelium may be seen through the interstices of the stroma in the outer half of the iris in a female aged 64 years with ocular hypertension.

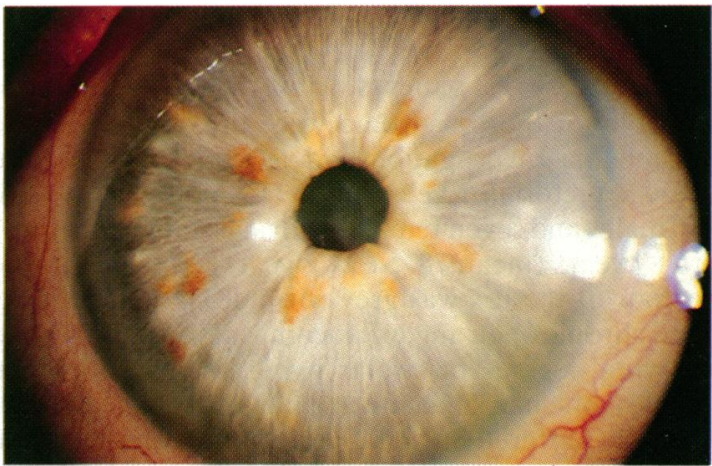

Fig. 4 'Senile tears' of the iris stroma in a female aged 83 years with chronic open angle glaucoma. These 'tears' have the appearance of multiple fine tears at the pupil margin due to small atrophic defects in the stroma. There is loss of the pigment ruff.

was usually present in the inner third of the iris but was more widespread in the patients with diffuse stromal atrophy. This finding was present to some degree in all cases.

3 . Gross patchy atrophy of the pigment epithelium in the region of, but less extensive than, the stromal atrophy in the inner third of the iris. This was present in three patients, who were not affected by herpes simplex or herpes zoster (Fig. 5).

4. Diffuse scatter of pigment granules through the iris stroma was seen in three patients.

On slit-lamp examination evidence of microneovascularisation of the iris was difficult to detect, but the following changes were noted: very fine vessels close to the pupil margin were seen in six patients; some radial vessels were seen in the atrophic inner third of the iris in five patients; microangiomata were

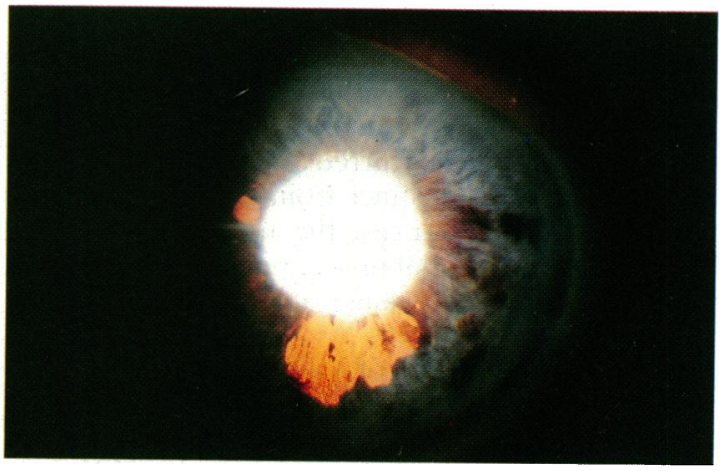

Fig. 5 Gross patchy atrophy of the pigment epithelium of the iris shown on retroillumination of the iris in the same eve as Fig. 2. This patient had not suffered previous herpes simplex or zoster in either eye. 


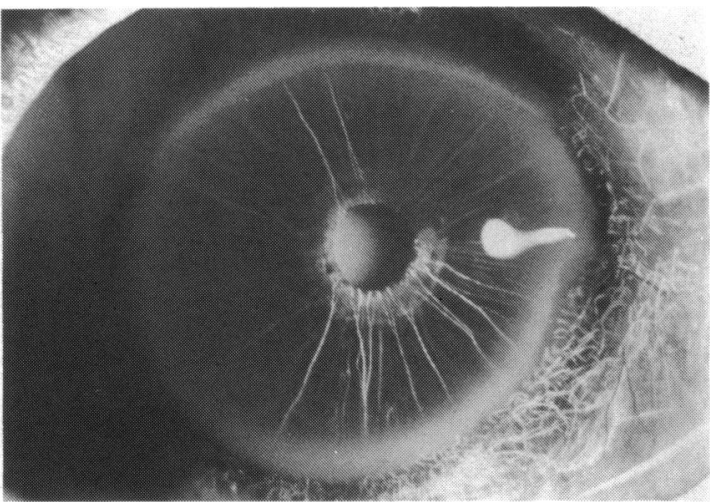

Fig. 6a Iris angiogram at 55 seconds in a male aged 84 years with diffuse iris atrophy and chronic open angle glaucoma. The very first dye appeared at 48 seconds. Marked segmental perfusion defects are still seen. There is early evidence of filling of the peripupillary plexus but there is still no venous filling. Nine definite arteries are demonstrated.

present at the depigmented pupil margin in three patients.

Fluorescein angiography of the iris showed abnormalities in all patients. There was some difficulty in differentiating arterial from venous filling, but we noted a change in the number and density of vessels which we considered to be a change from arterial to venous perfusion. The findings were as follows:

1. The appearance of dye was delayed, sometimes markedly, in all patients, appearing at a mean of 26 (SEM 3) seconds, range 15 to 51 seconds (Fig. 6a).

2. There was a long arteriovenous circulation time and sometimes venous filling was extremely slow or very difficult to determine precisely: mean arteriorvenous circulation time 11 (SEM 2) seconds, range 3 to 30 seconds (Fig. 6a).

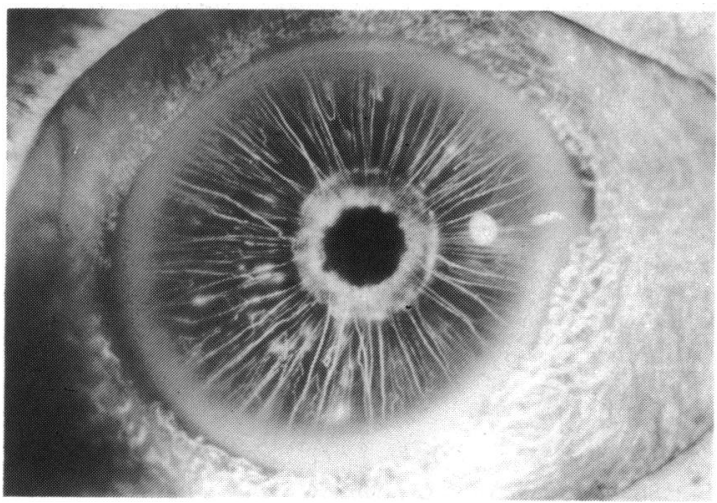

Fig. 6b At 76 seconds venous filling is established. There is a well marked peripupillary plexus which is leaking heavily and mid stromal leaking tufts are also seen.

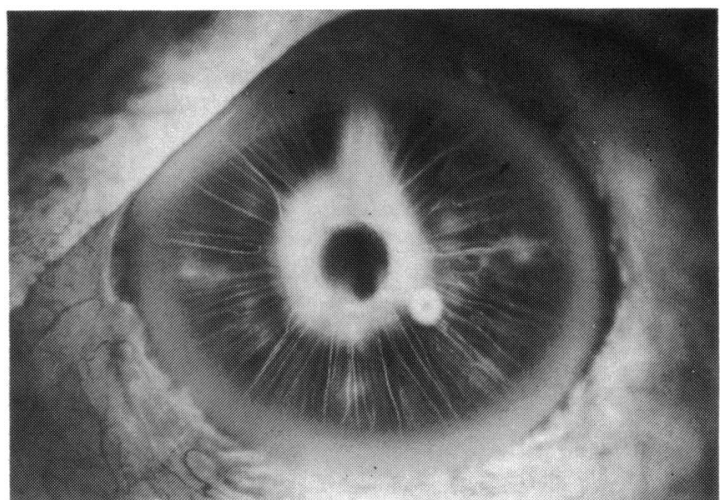

Fig. 6c - At 96 seconds there is a smoke stack effect from the leaking peripupillary plexus.

3. Hypoperfusion of the iris was a feature of all cases with $(a)$ a small number of arteries, always less than 30 (mean 17, SEM 1, range 10 to 28) (Figs. 6a, $7 \mathrm{a}) ;(b)$ the arteries were usually very thin and attenuated, though sometimes a few dilated radial vessels were present (Figs. 7a, 8a). The arterioles not infrequently appeared beaded (Fig. 9).

4. In 10 patients large sectors of iris appeared to be without arterial perfusion with late venous filling. In five of these almost half of the iris appeared to have very little arterial supply (Figs. 6a, 7a).

5. Leakage of dye from the pupil margin occurred in all cases and was often quite marked, with a smoke stack effect in late pictures due to upward circulation of the dye (Figs. 7b, 7c, 10, and 11).

6. Peripupillary neovascularisation was distinct from pupillary leakage and was present in 16 cases (Figs. 6c, 11). There was a fine dense plexus of

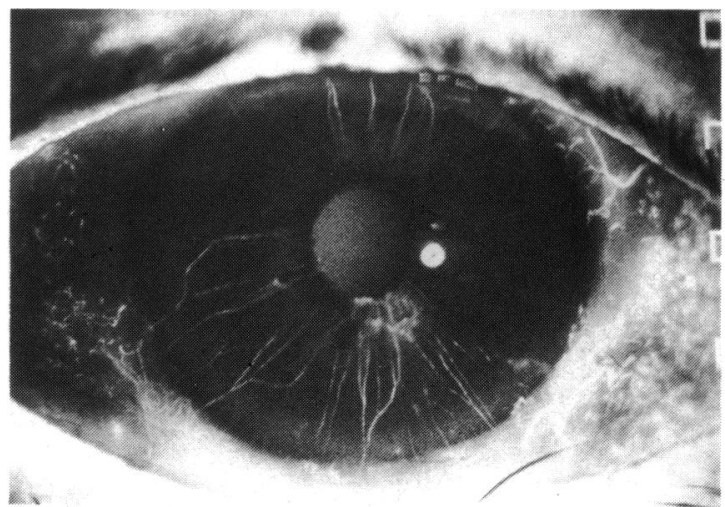

Fig. 7a Iris angiogram at 26 seconds of the same patient as Fig. 3 with diffuse iris atrophy and ocular hypertension. The first dye appeared at 22 seconds. At 26 seconds the venous phase is still not established. There are marked sectorial filling defects from 2 to 4 and 9 to 11 with thin attenuated arteries. Early leakage is occurring in the mid stroma. 


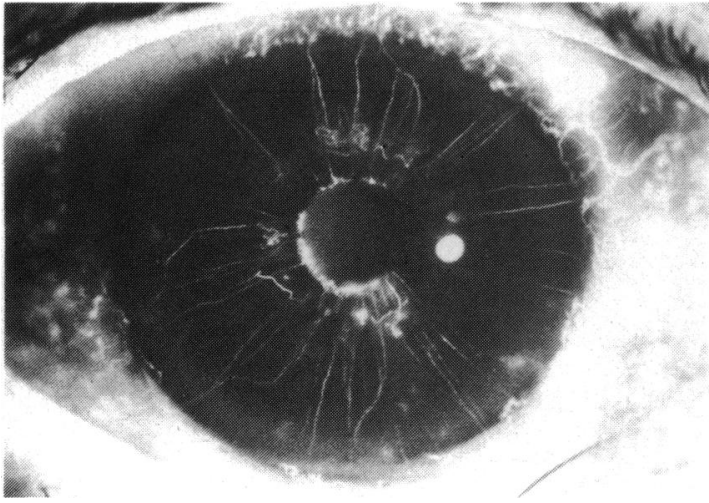

Fig. 7b At 33 seconds some sectorial defects in perfusion are still present. Complex neovascularisation is seen in the inner third of the stroma. Pupillary leakage is prominent.

leaking vessels adjacent to the pupil margin but not extending as far as the lesser vascular circle of the iris. The leakage was very heavy in some cases, again causing a smoke stack effect in the late pictures (Figs. $6 b, 8 b)$.

7. In addition, leaking tufts or vascular nodules occurred elsewhere in the stroma in eight patients. These were not usually numerous and were most common in the region of the lesser circle of the iris (Figs. 6b, 8b).

8. In six patients more extensive and complex neovascularisation was present elsewhere in the stroma with heavy leakage (Figs. 7b, 7c, 8b).

9. An expanded and prominent lesser vascular circle of the iris was present in nine patients. In these the iris atrophy affected the inner third of the iris, so that the lesser circle was much further from the pupil margin than normal. This prominent lesser circle did

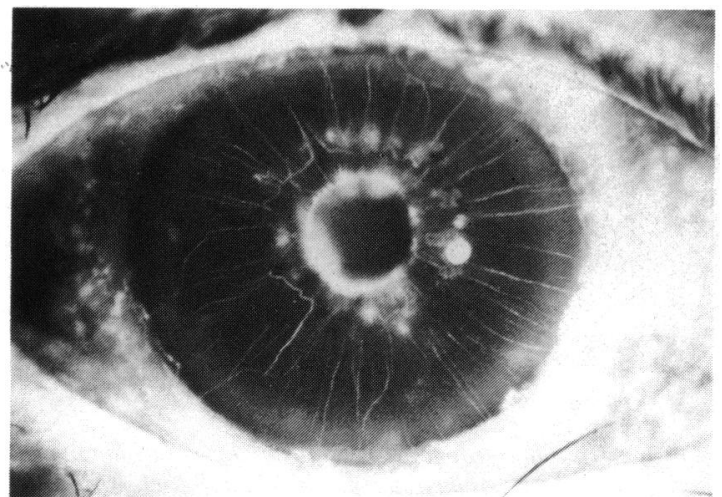

Fig. 7c At 50 seconds all sectors of the iris are filling in the venous phase. There is heavy peripupillary leakage from complex new vessels in the inner third of the iris stroma with commencing smoke stack effect.

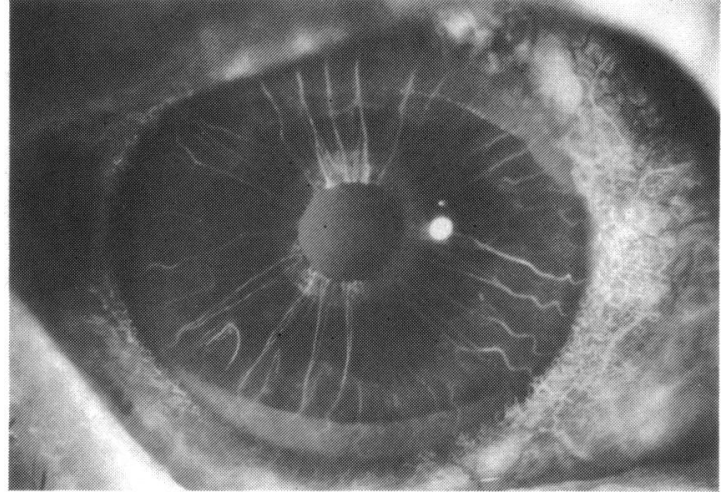

Fig. 8a Iris angiogram of a female aged 78 years with atrophy of the inner third of the stroma and ocular hypertension. The first dye appeared at 17 seconds. At 20 seconds there is arterial perfusion of thin attenuated arteries. There is very early filling of the peripupillary plexus. Vessels of the expanded lesser circle are starting to fill.

not leak, suggesting that it was due to dilatation of pre-existing anastomoses and filled before the venous flush, after the initial arterial filling. Iris atrophy was often not present in the upper third of the iris, and then the lesser circle was at the normal distance from the pupil margin. The arterial perfusion of the iris was usually much better in the upper third in such cases (Figs. 8b, 11).

10. Peripheral vascular loops were present and prominent in eight patients. These did not reach the inner iris and appeared as loops which perfused early, did not leak and were not associated with venous drainage (Fig. 9).

11. Arterial beading was present in six cases (Fig. 9).

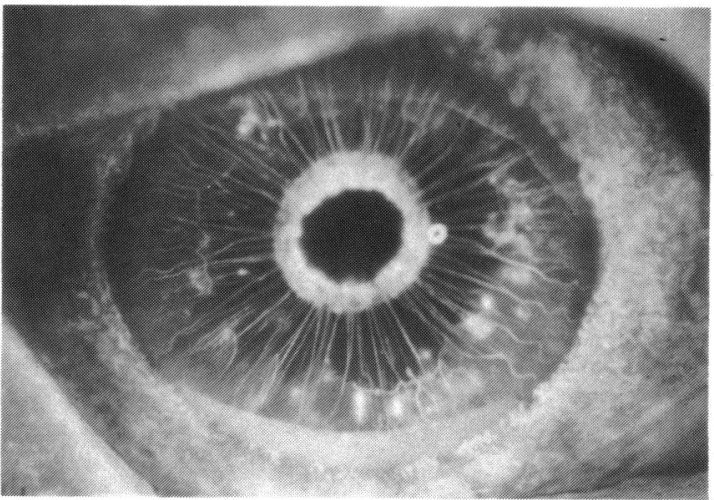

Fig. 8b At.37seconds, in the venous phase, numerous tufts are seen in the region of the lesser vascular circle of the iris. There is considerable leak from the peripupillary venous plexus. 


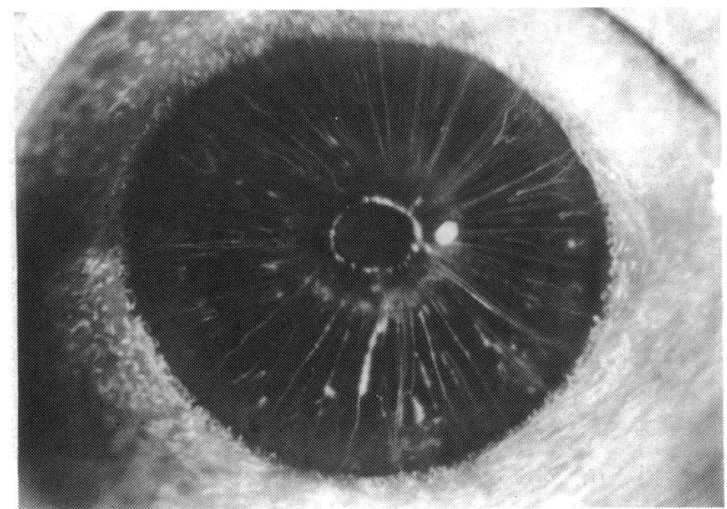

Fig. 9 Iris angiogram of a male aged 83 years with 'senile tears' of the iris and ocular hypertension. At 34 seconds there are prominent peripheral loops with beading of the vessels.

The neovascular changes were related to the iris atrophy in the following ways: The expanded lesser circle was related to the commonly occurring atrophy of the inner third of the iris. Although segmental hypoperfusion of the iris did not appear to be closely related to segmental atrophy of the iris, atrophy was uncommon superiorly and arterial perfusion was usually best in this area. In some cases where iris atrophy was particularly marked there was a heavy leakage of dye in the area of the atrophy, probably from damaged, compromised, or exposed radial vessels in the area.

There was a relationship between the iris changes and raised intraocular pressure. Most of these patients presented for investigation of raised intraocular pressure. Eight patients were under treatment for chronic open angle glaucoma with raised intraocular pressure. Seven patients were seen with

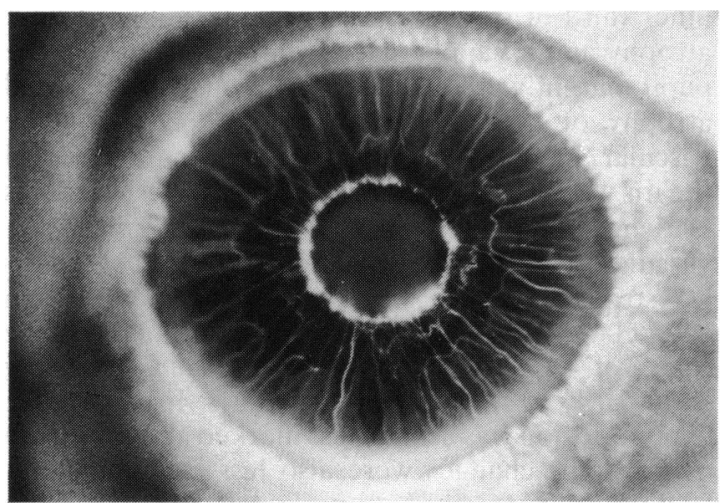

Fig. 10 Iris angiogram in female aged 86 years with atrophy of the inner third of the iris and chronic open angle glaucoma. The first dye appeared at 20 seconds. At 40 seconds there is well marked pupillary leak and well developed lesser circle of the iris. The pupillary leak is showing a smoke stack effect.

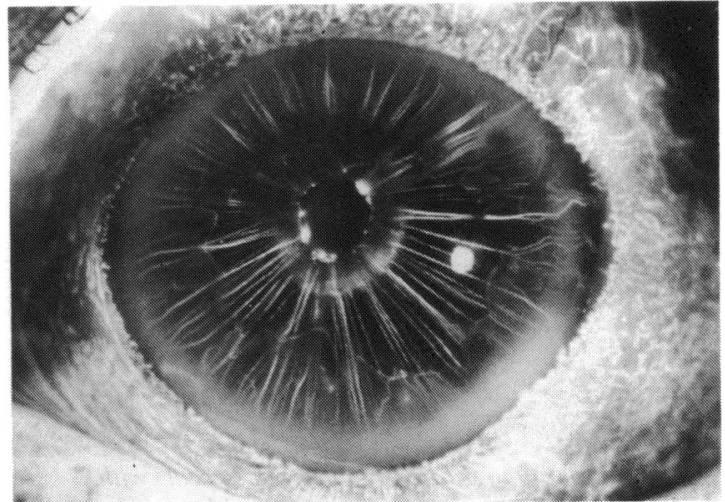

Fig. 11 Iris angiogram in same eye as Fig. 1. The first dye appeared at 20 seconds. At 35 seconds (venous phase) an expanded lesser vascular circle is well shown with peripupillary leakage of dye.

elevated intraocular pressures in the absence of both pathological cupping of the optic disc and of field loss. One patient was seen with typical subacute angle closure glaucoma but had not developed an acute attack. In this patient the iris atrophy was not typical of acute glaucoma but rather of the condition which we are describing. In one eye there was no rise in intraocular pressure, and this is the only case which we have seen with the findings typical of the condition in the absence of raised intraocular pressure.

On gonioscopy all angles were wide and open all round except for the case of subacute angle closure glaucoma. In most patients trabecular pigmentation was recorded as moderate, but in three it was light and in two heavy. The iris processes were scanty in all but two cases, in which the iris processes were fine, moderate in number, and extended to the trabecular meshwork. The slit-lamp evidence of microneovascularisation was very subtle, and no fine vascular changes were noted in the angle on gonioscopy.

Specular microscopy showed a significant decrease in endothelial cell counts in the patients with iris atrophy compared with age matched normal controls (mean 2202, SEM 63/ $\mathrm{mm}^{2}$ in iris atrophy; mean 2887 , SEM $90 / \mathrm{mm}^{2}$ in the controls; $\mathrm{p}<0.01$ Wilcoxon matched-pairs signed-rank test). There was an increase in pleomorphism and polymegathism of the endothelial cells in the patients with iris atrophy.

Arterial hypertension requiring medication was present in four patients and ischaemic heart disease requiring medication was present in two. The remainder were in good general health, and neither diabetes mellitus nor chronic respiratory disease was present in any of the patients.

Digital subtraction angiography showed no significant abnormality in six cases with abnormalities in 


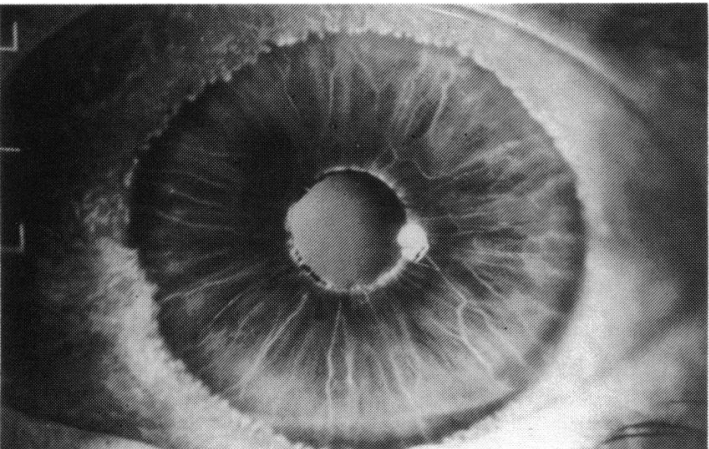

Fig. 12a Iris angiogram in female aged 74 years with normal grey iris. The first dye appeared at 21 seconds. At 48 seconds there is good perfusion of the iris, slight pupillary leak only and no other evidence of microneovascularisation nor expanded lesser circle.

four cases. There was $50 \%$ to $60 \%$ stenosis of the origins of both internal carotid arteries in the 83-yearold male with 'senile tears' of the iris and ocular hypertension. A $50 \%$ stenosis of the right internal carotid artery origin, with a lesser narrowing of the external carotid origin and a haemodynamically insignificant plaque at the origin of the left internal carotid artery, was present in the 85-year-old female with atrophy of the inner third of the iris and chronic open angle glaucoma. In this patient the iris atrophy was less on the side of the significant carotid stenosis. In the male aged 73 years, with atrophy of the inner third of the iris and ocular hypertension, there was a mild but insignificant narrowing of the origin of the right internal carotid artery, and once again the iris atrophy was more marked on the contralateral side with no stenosis. Finaly, in the male aged 75 years with diffuse bilateral iris atrophy and chronic open angle glaucoma there was a very slight irregularity at, and just distal to, the origin of the right internal carotid artery, but no disease was demonstrated in the left carotid system.

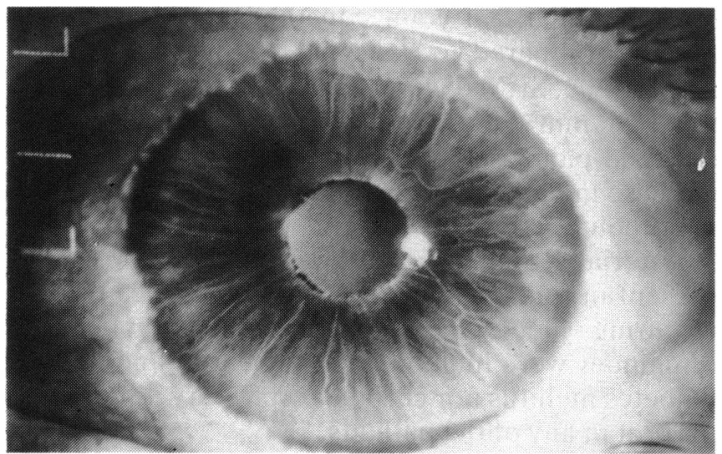

Fig. 12b At 96 seconds perfusion is fading and there is still no significant leakage. The pupillary ruff is well seen.

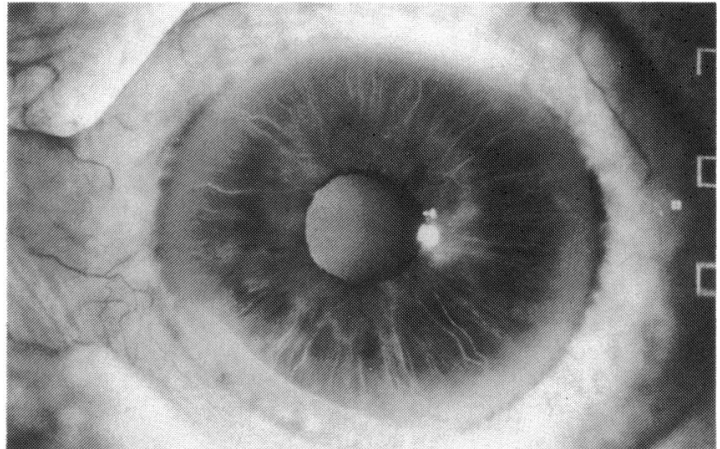

Fig. 13 Iris angiogram in female aged 85 years with normal grey iris. The first dye appeared at 18 seconds. Late picture at 121 seconds showing no significant leakage of dye. Note the clarity of the pupillary ruff.

On fluorescein angiography of the normal group the mean arterial time was mean 20 (SEM 2) seconds, range 14 to 27 seconds. The eyes were well perfused with a normal number of radial arterioles of good calibre, though a small area of sectorial hypoperfusion was present in one patient. Six patients showed some pupillary leak, though this was slight except in one patient where it was moderate. No patient showed peripupillary neovascularisation nor expanded lesser circle. One patient showed one leaking tuft. There was no arterial beading and no neovascular plexuses had developed (Figs. 12a, 12b, 13).

\section{Discussion}

This group of patients is distinct enough to form a clinical entity. The atrophy of the iris stroma was bilateral and most commonly patchy affecting the inner third of the lower iris stroma, though diffuse atrophy and an appearance of local notching of the pupil margin were also seen. Although the stromal atrophy of the iris varied in its appearance, the vascular changes found on fluorescein angiography of the iris were similar throughout the group. There was evidence of hypoperfusion of the iris and microneovascularisation. The arterial hypoperfusion of the iris was striking in degree, being much more marked than age related changes, and we believe that it is the cause of the iris changes, rather than occurring secondarily to the atrophy of the iris. In particular, hypoperfusion was usually less marked above and the iris atrophic changes were also less frequent here. Sectorial defects in hypoperfusion, distinct from physiological delay in filling described by Hayreh and Scott, 2" together with a delayed arteriovenous circulation time also support hypoperfusion as the cause of iris atrophy. 
It is possible that the vascular changes are secondary to a rise in intraocular pressure, but we have seen the condition in the absence of raised intraocular pressure. In some cases with less atrophy of the iris the ocular hypertension was relatively mild, suggesting a progressive process which may go on to a more extreme rise in intraocular pressure as the process advances. Thus the ocular hypertension appears to be secondary to the iris changes. Further, six patients had received no previous treatment for their ocular hypertension, so it is unlikely that the vascular changes were secondary to glaucoma medication.

The iris microneovascularisation was a subtle change which had previously been noted among the vascular changes in anterior segment pigment dispersal syndrome, which was thought to be due to a congenital mesodermal defect. ${ }^{12}$ This iris microneovascularisation was much less marked than the gross neovascularisation of the iris which occurs in neovascular glaucoma following previous central retinal vein occlusion, but its pathogenesis may be similar, though in the cases we describe the iris atrophy arose as a result of more gradual and more localised ischaemia than in central retinal vein occlusion. Clinically, the iris microneovascularisation is difficult to detect, but fine vessels may be seen close to the pupil margin, from which the pupillary ruff is usually lost, as. in the loss of pupillary ruff previously reported in pseudoexfoliation of the lens capsule. ${ }^{1: 14}$ Fine radial vessels may also be seen with the slit-lamp high power, but these are probably normal radial vessels which do not leak. The microangiomata are similar to the vascular tufts at the pupil margin described by Cobb. ${ }^{\prime \prime}$

The pupillary leakage of dye which was seen in all cases was probably due to neovascular tufts occurring at the pupil margin and was much more marked than that seen in some elderly patients. The heavy pupillary leakage from fine vessels may be either from fine neovascularisation or from damaged vessels of the capillary plexus which is normally present in this location. The sphincter muscle, which is supplied by the capillary plexus, remained intact. The expanded prominent lesser circle of the iris appeared to be formed by opening up of a pre-existing arterial anastomosis, and this became displaced outwards with loss of the inner third of the stroma. The leaking tufts in the iris stroma may be consistent with early neovascularisation, ultimately developing into the more complex systems of neovascularisation sometimes seen in the stroma on angiography. The beading of the arterioles is consistent with changes occurring as a result of damage to the vessel walls.

The most typical atrophy of the iris stroma affected the inner third of the stroma, often being patchy in distribution, with sparing above. This was accom- panied by some loss of the pigment epithelium and is consistent with this inner part of the iris being more susceptible to the effects of hypoperfusion occurring in later life. Anterior segment pigment dispersal syndrome is also associated with a relative hypoperfusion of the iris but this is probably present from birth, with some degree of hypoplasia of the iris stroma. This results in an instability of the pigment epithelium which is easily shed in a patchy manner in the region of the dilator muscle in susceptible individuals.

Patients in the current series were elderly, and, although some were under treatment for arterial hypertension or ischaemic heart disease, most were in good general health for their age, with no chronic respiratory disease or other cause of peripheral hypoxia which might predispose to pupillary leakage ${ }^{22}$ or other changes. Although carotid obstruction has been reported in ischaemic ocular inflammation, ${ }^{19}$ digital subtraction angiography failed to show a significant abnormality in most patients, excluding subclinical carotid insufficiency with hypoperfusion of the head and neck as a major aetiological factor in this condition.

The relationship of the iris atrophy in this series to glaucoma may be partly due to pigment release, most of the angles showing marked or moderate pigmentation of the trabecular meshwork. Iris processes were not a prominent feature of this condition, as they often are in various forms of iris hypoplasia and anterior segment pigment dispersal syndrome. ${ }^{23}$ However, the hypoperfusion which causes the iris atrophy may also affect the trabecular mesh, causing ischaemic changes which result in an increased resistance to aqueous outflow. The less marked degree of iris atrophy often seen in chronic open angle glaucoma suggests that this may be a common mechanism in the production of increased resistance to aqueous outflow. The more permeable iris capillaries may also affect aqueous dynamics by allowing leakage of plasma components into the aqueous.

Diffuse atrophy of the iris stroma occurred less commonly than atrophy of the inner third of the stroma, but was accompanied by similar vascular changes and ocular hypertension. Diffuse reflux of light through the pigment epithelium was also a feature of these cases.

'Senile tears' of the iris gave an appearance of tears at the pupil margin but were bilateral, with no other suggestion of contusion injury. They appear to be due to local atrophy of the iris stroma close to the pupil. Hypoperfusion of the iris was present in these cases with prominent microneovascular changes and segmental hypoperfusion; this hypoperfusion may be a factor in producing this local stromal change.

The changes in iris stroma which we have described 
were much more marked than any senile changes which we have previously observed and constitute a distinct disease entity. Although the changes were expressed in several different ways, which we have described, the underlying vascular changes were essentially similar in all, and much more marked than senile vascular changes. The findings in the comparable group of elderly patients with normal irides which are described here demonstrate that, although some slight changes in perfusion and a little pupillary leakage may occur in otherwise normal elderly patients, they are only minor in degree, and the abnormal changes which we have described are pathological in nature.

Kottow $^{22}$ has remarked that although some vascular abnormality may be seen on fluorescein angiography in elderly patients it is difficult to be sure whether even these mild changes are really age related or pathological. The vascular changes were different from the progressive midstromal changes we have observed in cyclitis ${ }^{24}$ and usually much less florid than the changes previously described in pseudoexfoliation of the lens capsule. ${ }^{\text {Is }}$

The bilaterality of the changes suggests that the iris atrophy is an abiotrophy appearing in later life, associated with endothelial cell changes. These changes are not as marked as those in Fuchs' endothelial dystrophy, which Bahn et al. ${ }^{\text {i5 }}$ have considered to be due to an endothelial dystrophy. The vascular hypoperfusion common to all cases suggests that this is the causal mechanism, but why these small arteries should fail in later life is not clear. This condition does not appear to be part of a generalised vascular disease but rather is a local defect in the arterial circulation of the iris. Iridoschisis and some forms of the ICE syndrome are other iris conditions which also present later in life, but they are quite different in appearance from the entity which we describe.

There were seven patients with ocular hypertension only, without cupping of the optic discs. The absence of optic disc damage in the presence of hypoperfusion of the iris suggests that there is no associated hypoperfusion of the posterior segment of the eye, which if present would render the optic disc more susceptible to the effects of ocular hypertension.

It is striking that all patients had grey (or blue) irides. Although hypothetical, this suggests that these irides are far more susceptible to the effects of this hypoperfusion. A further factor in the development of atrophy may be a greater susceptibility of light coloured irides to the effects of solar radiation. The higher incidence of iris atrophy in males in this series may relate to their increased exposure to such solar radiation. The sparing of the upper third of the iris stroma which is usually shielded by the upper lid also suggests that exposure is a factor in the aetiology of this condition.

In conclusion, atrophy of the iris due to hypoperfusion with microneovascularisation as we have described is a distinct clinical entity, occurring moderately frequently in the elderly. It is a local condition of the iris and may be diagnosed on slitlamp examination, with confirmation of hypoperfusion by fluorescein iris angiography. It is commonly, but not invariably, associated with glaucoma.

This work was carried out under Research Projects 20 and 92 of the Royal Victorian Eyc and Ear Hospital. We thank the Department of Medical Illustration for the invaluable assistance with the clinical photographs and iris angiograms.

\section{References}

1 Hoskins HD Jr, Shaffer RN, Hetherington J. Anatomical classification of the developmental glaucomas. Arch Ophthalmol 1984; 102: 1331-6.

2 Weatherill JR. Hart CT. Familial hypoplasia of the iris stroma associated with glaucoma. BrJ Ophthalmol 1969; 53: 433-8.

3 Gillies WE. Hypoplasia of the iris stroma in Gregg's syndrome unaccompanied by cataract but with deafness, rubella retinopathy and onset of glaucoma in adult or adolescent life. Aust $J$ Ophthalmol 1980); 8: 189-92.

4 Grant WM. Walton DS. Progressive changes in the angle in congenital aniridia, with development of glaucoma. Am J Ophthalmol 1974; 78: 842-7.

5 Alkemade PPH. Dysgenesis mesodermalis of the iris and the cornea. A study of Reiger's syndrome and Peters' anomaly. Assen: Van Gorcum, 1969: 44-5.

6 Polack FM, Graue EL. Scanning electron microscopy of congenital corneal leukomas (Peters' anomaly). Am J Ophthalmol 1979; 88: $169-78$.

7 Heath P. Essential atrophy of the iris. Am J Ophthalmol 1954: 37: 219-34.

8 Campbell DG. Shiclds MB. Smith TR. The corneal endothelium and the spectrum of essential iris atrophy. Am J Ophthalmol 1978; 86: 317-24.

9 Chandler PA. Atrophy of the stroma of the iris. Endothelial dystrophy, corneal edema, and glaucoma. Am J Ophthalmol 1956; 41: 607-15

10 Hirst LW, Quiglcy HA, Stark WJ. Specular microscopy of iridocorneal endothelia syndrome. Am J Ophthalmol 1980; 89: $11-21$

11 Mills PV. Iridoschisis. BrJ Ophthalmol 1967: 51: 158-64.

12 Gillies WE. Tangas $C$. Fluorescein angiography of the iris in anterior segment pigment dispersal syndrome. BrJ Ophthalmol 1986; 70: 284-9.

13 Vannas A. Fluorescein angiography of the vessels of the iris in pseudoexfoliation of the lens capsule, capsular glaucoma and some other forms of glaucoma. Acta Ophthalmol (Kbh) 1969; 105 (suppl): 9-75.

14 Vannas A. Vascular changes in pseudoexfoliation of the lens capsule and capsular glaucoma. A fluorescein angiographic and electron microscopic study. Graefes Arch Klin Exp Ophthalmol 1972; 184: 248-53.

15 Brooks AMV, Gillics WE. Fluorescein angiography and fluorophotometry of the iris in pseudoexfoliation of the lens capsule. $\mathrm{Br}$ J Ophthalmol 1983: 67: 249-54.

16 Fuchs E. Ueber komplikationen der heterochromic. $Z$ Augenheilkd 1906: 15: 191-212.

17 Kimura SJ, Hogan MJ, Thygeson P. Fuchs' syndrome of heterochromic cyclitis. Arch Ophthalmol 1955; 53: 179-86. 
18 Winstanley J. Iris atrophy in primary glaucoma. Trans Ophthalmol Soc UK 1961: 81: 23-38.

19 Jacobs NA. Ridgway AEA. Syndrome of ischacmic ocular inflammation: six cases and a review. BrJ Ophthalmol 1985; 69: 681-7.

20 Hayreh SS, Scott WE. Fluorescein iris angiography. I. Normal pattern. Arch Ophthalmol 1978; 96: 1383-9.

21 Cobb B. Vascular tufts at the pupillary margin: a preliminary report on 44 patients. Trans Ophthalmol Soc UK 1968; 88: 211-21.
22 Kottow MH. Anterior segment fluorescein angiography. Baltimore: Williams and Wilkins, 1978: 51 .

23 Lichter PR. Shaffer RN. Iris processes and glaucoma. Am J Ophthalmol 1970) 70: 905-11.

24 Brooks AMV, Grant G, Gillies WE. Changes in the iris vasculature and corneal endothelium in chronic cyclitis. Aust NZ J Ophthalmol 1986; 14: 189-97.

25 Bahn CF. Falls HF, Varley GA, Meyer RF, Edelhauser HF, Bourne WM. Classification of corneal endothelial disorders based on neural crest origin. Ophthalmology 1984; 91: 558-63.

Accepted for publication 26 September 1986. 